\title{
Effect of Aerobic Training During Pregnancy on Seizure-Induced Depression- in Mouse
}

\author{
Ayoob Sabaghi ${ }^{1}$, Ali Heyrani ${ }^{*}$, Amir Kiani ${ }^{2}$, Namdar Yousofvand ${ }^{3}$ \\ ${ }^{1}$ Faculty of Physical Education and Sport Sciences, Razi University, Kermanshah, Iran \\ ${ }^{2}$ Faculty of Pharmacology, Kermanshah University of Medical Sciences, Kermanshah, Iran \\ ${ }^{3}$ Faculty of Sciences, Razi University, Kermanshah, Iran
}

Article Info:

\section{A BSTRACT}

Introduction: Depression during pregnancy is not uncommon. In addition, depression is more common in patients suffering from epilepsy. Aerobic activity has been reported to alleviate depression. The purpose of this study was to investigate the effect of aerobic training during pregnancy on seizure-induced depression. Materials and Methods: Pregnant mice were randomly divided to control, sham, and experiment groups. The pentylenetetrazol (PTZ) kindling was induced on days 14 to19 after pregnancy in the experiment group. Experiment group was divided to mice with and without aerobic activities. Aerobic activity was performed every day during pregnancy. The rate of depression in pregnant mice was measured between the $14^{\text {th }}$ and $19^{\text {th }}$ days of pregnancy once in 48 hours and two hours after PTZ injection. Results: Treatment with aerobic training significantly decreased seizureinduced depression as well as seizure severity in pregnant mice. Conclusion: The present s tudy showed that aerobic training during pregnancy is positively associated with decreasing depression and severity of seizure in PTZ-treated mice.

*Corresponding Author: Ali Heyrani

E-mail: iliaheirani2004@gmail.com 
تأثير تمرين هوازى در دوران باردارى بر ميزان افسردگى القا شدهُ ناشى از تشنج در موش سورى

\author{
ايوب صباغى'، على حيرانى "'، امير كيانى'، نامدار يوسفوند" \\ 'دانشكدة تربيت بدنى و علوم ورزشى، دانشكاه رازى، كرمانشاه، ايران

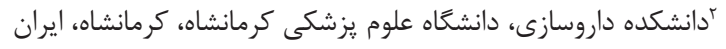

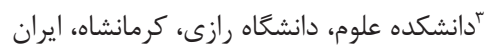

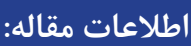

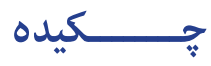

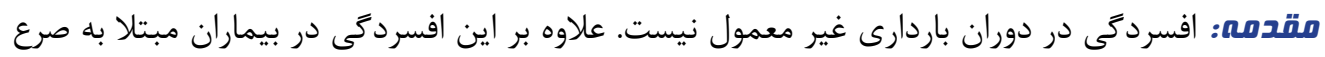

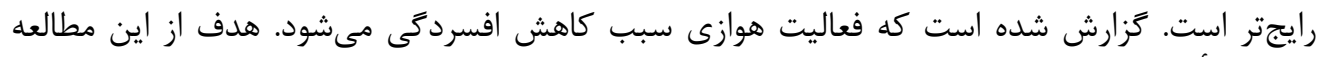

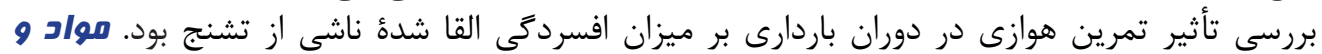

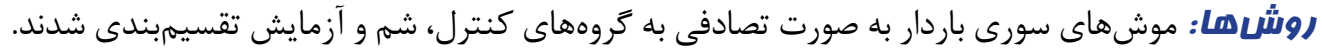

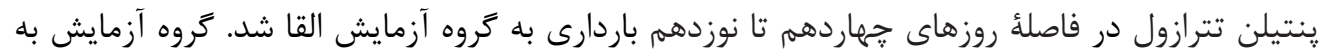

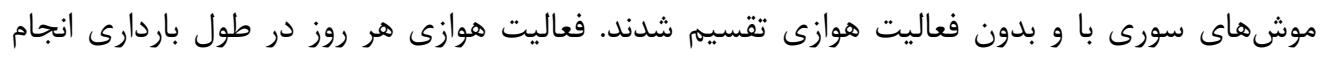

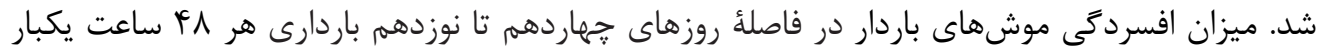

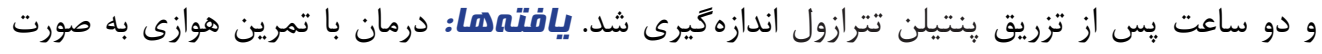

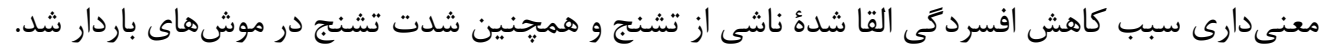

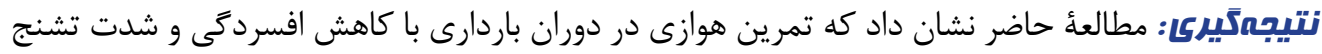

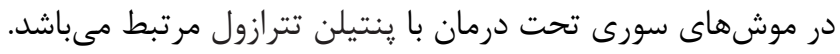

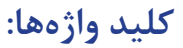

1. إنمرين r. باردارى

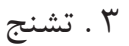

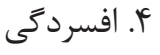

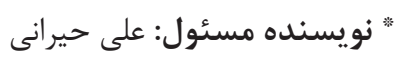
آدرس الكترونيكى: iliaheirani2004@gmail.com 


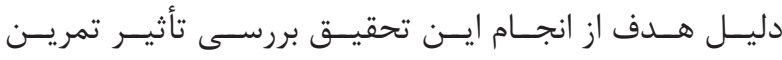

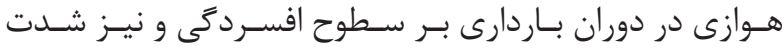

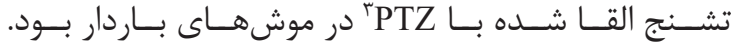

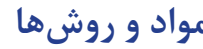

نمونها و شرايط نكَهدارى حيوانات

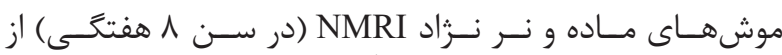

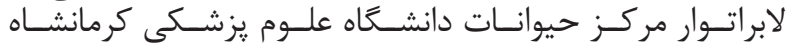

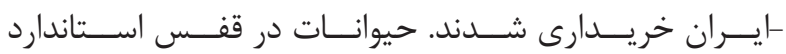

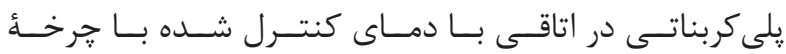

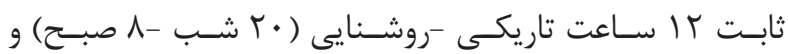

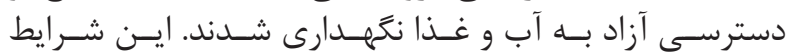

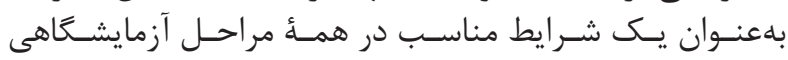

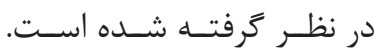
فرايند كيندل كردن

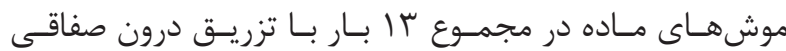

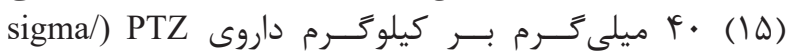

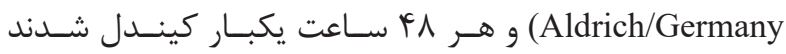

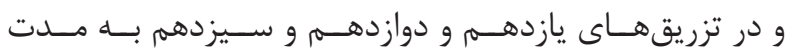

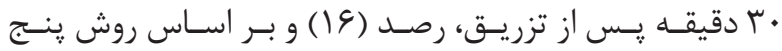

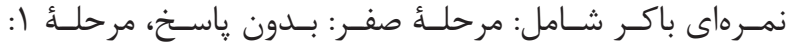

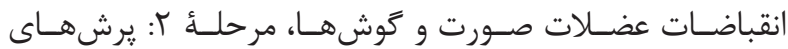

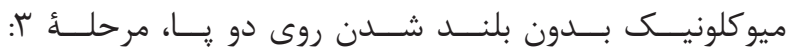

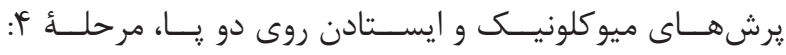

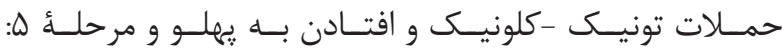

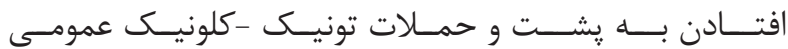

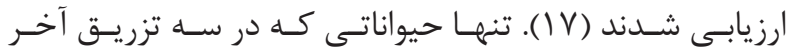

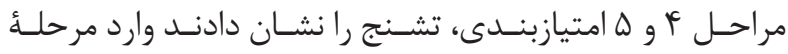

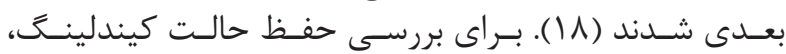

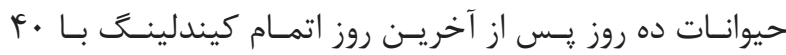

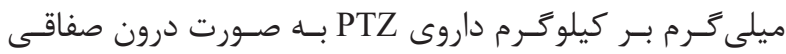

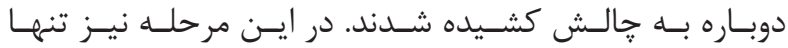

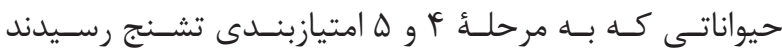

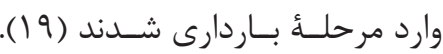

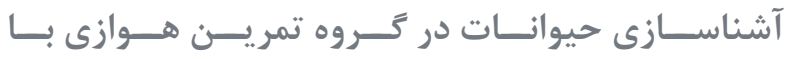
تردميــل المناسل

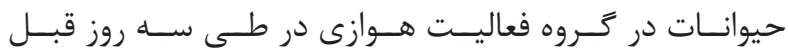

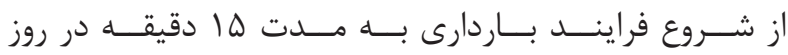

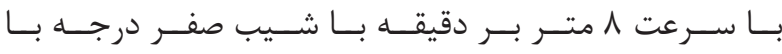

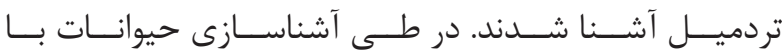

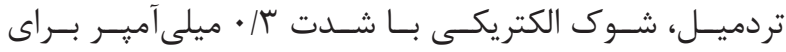

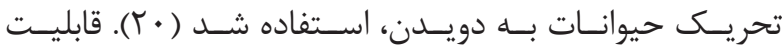

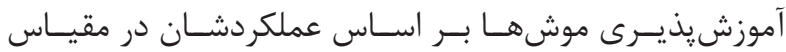

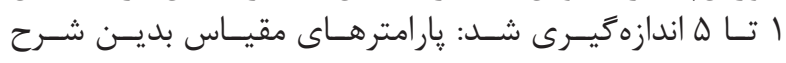

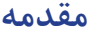

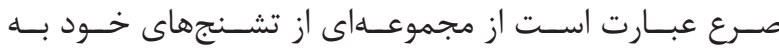

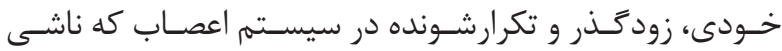

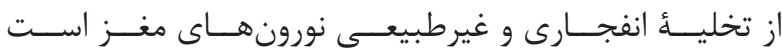

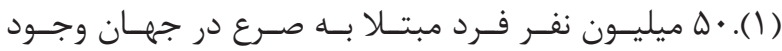

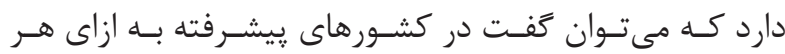

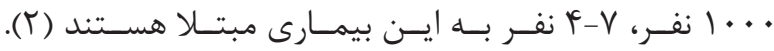

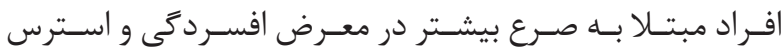

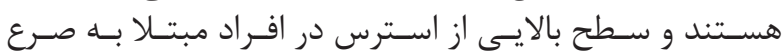

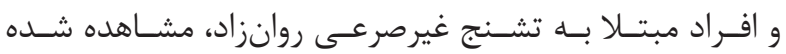

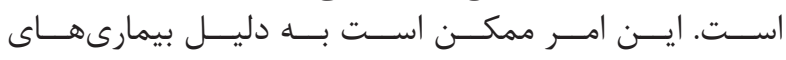

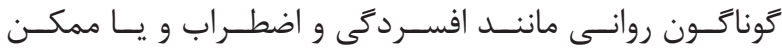

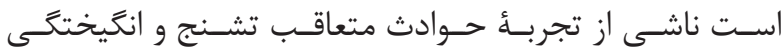

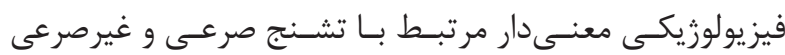

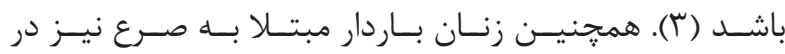

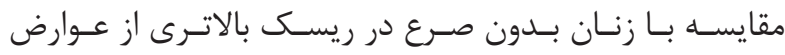

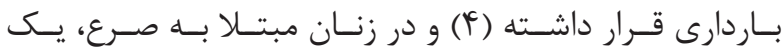

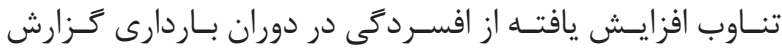

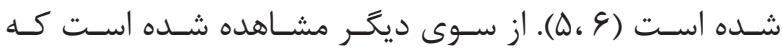

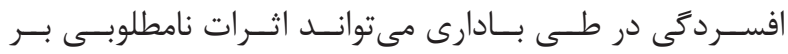

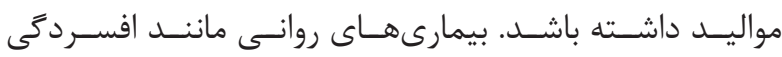

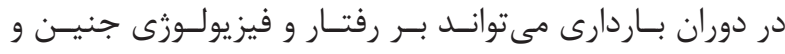

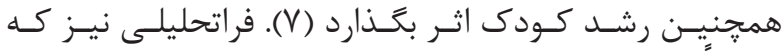

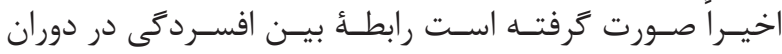

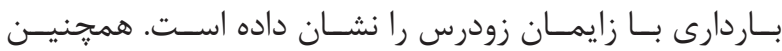

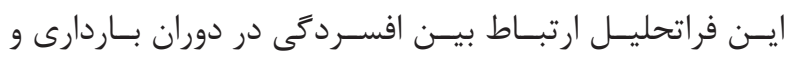

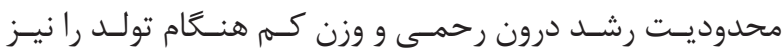

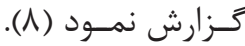

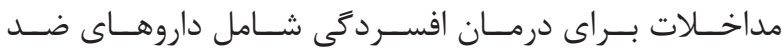

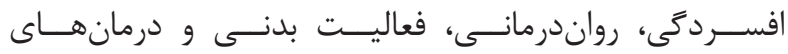

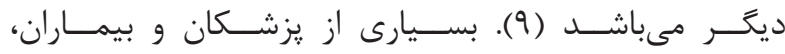

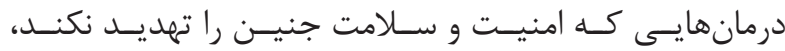

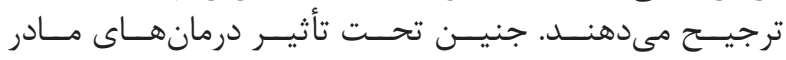

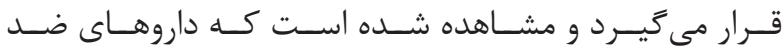

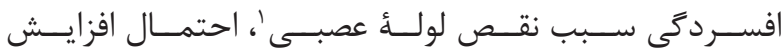

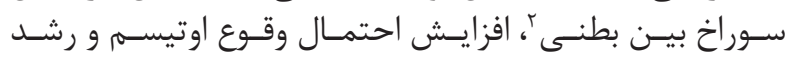

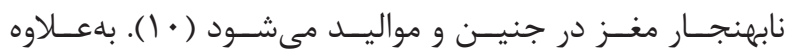

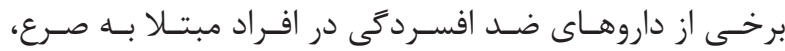

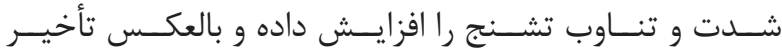

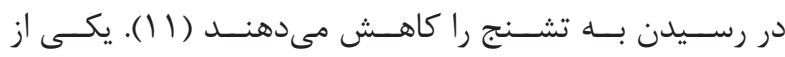

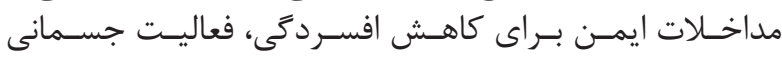

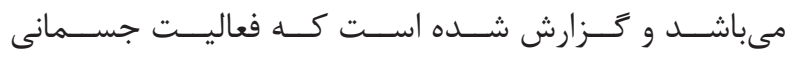

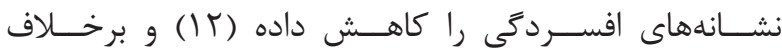

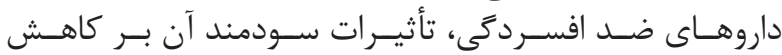

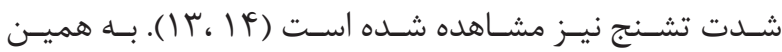

${ }^{1}$ Neural tube defects

${ }^{2}$ Ventricular septal defect

${ }^{3}$ Pentylenetetrazol 


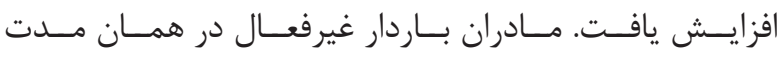

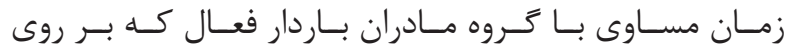

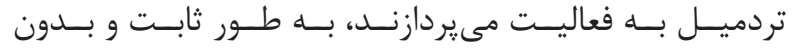

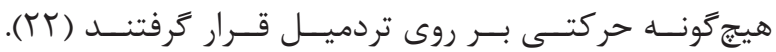

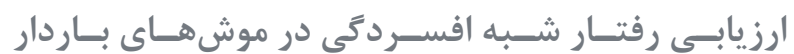

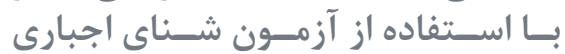

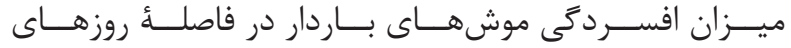

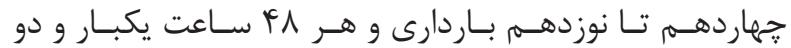

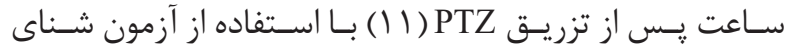

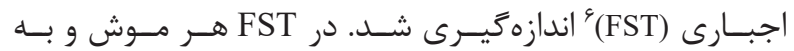

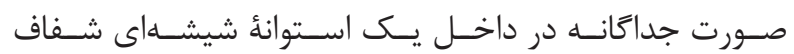

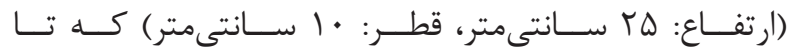

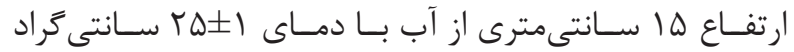

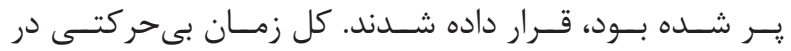

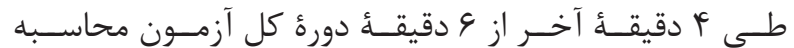

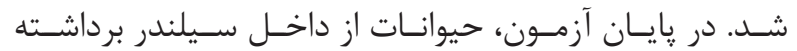

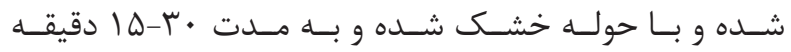

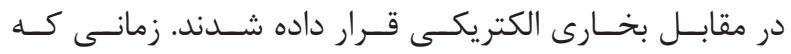

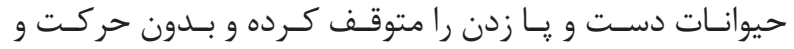

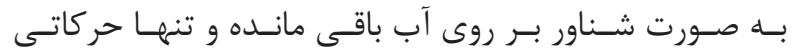

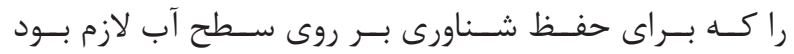

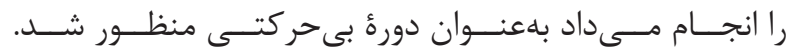

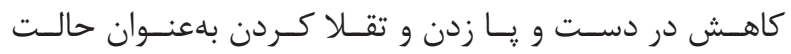

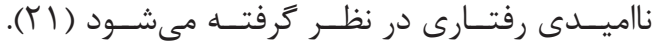

$$
\text { آناليز آمارى نامى رفتر }
$$

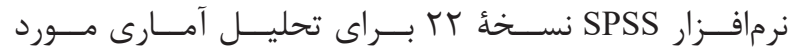

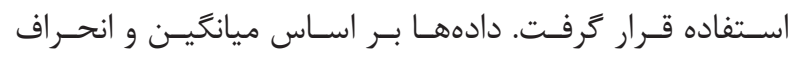

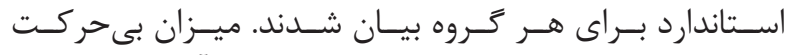

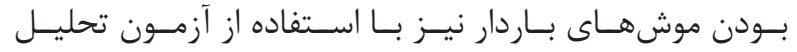

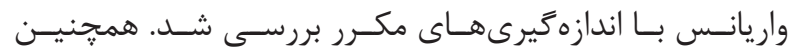

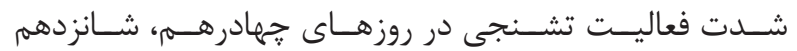

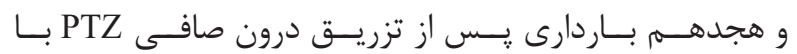

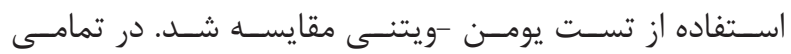

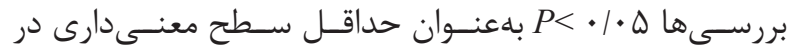

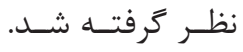

يافتهها

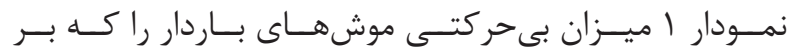

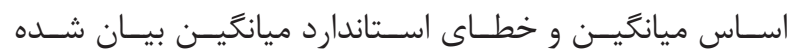

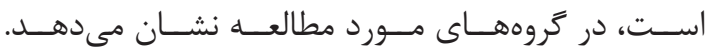

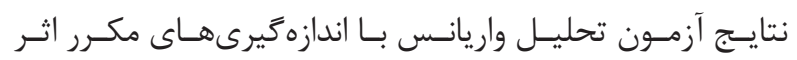

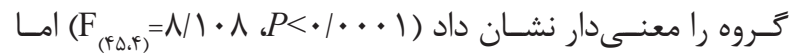
اثر زمـان (F) (F)

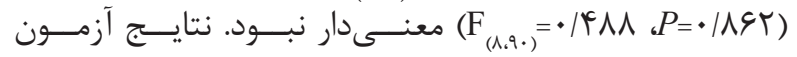

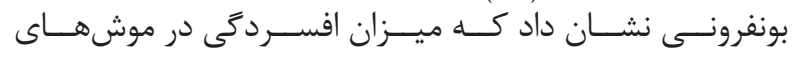

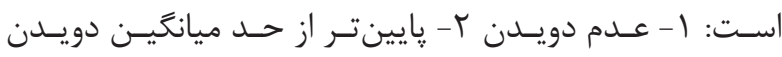

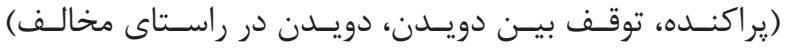

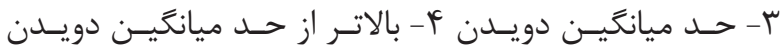

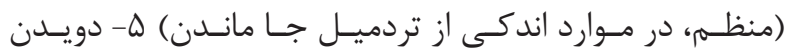

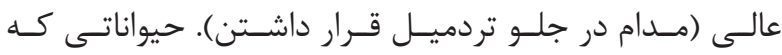

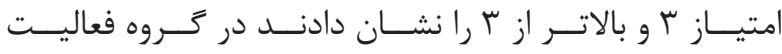

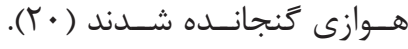

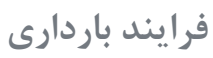

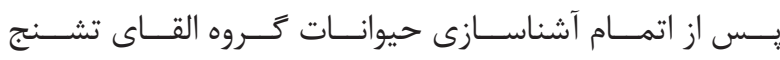

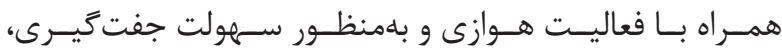

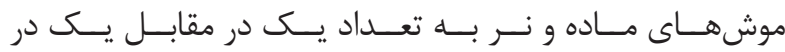

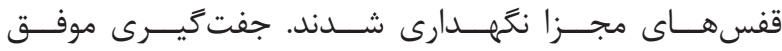

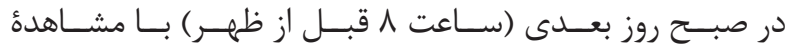

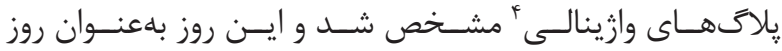

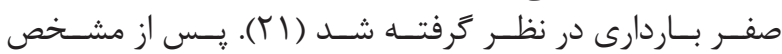

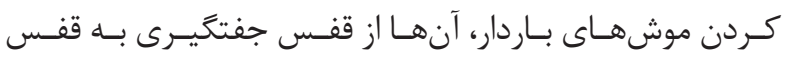

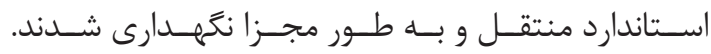

القاى تشنج در دوران باردارى

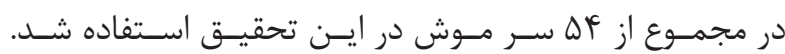

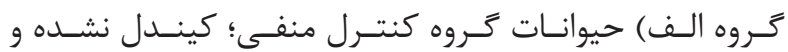

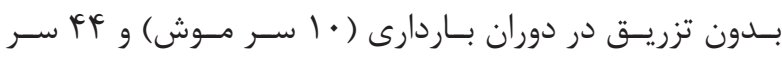

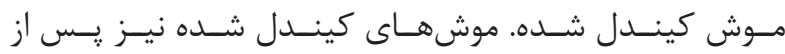

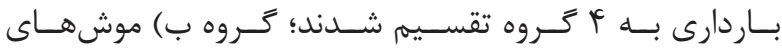

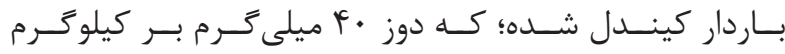

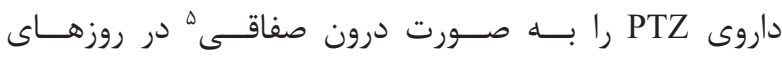

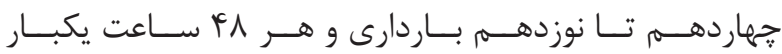

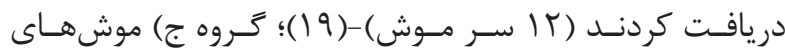

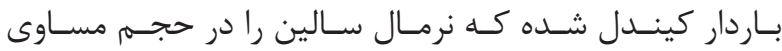

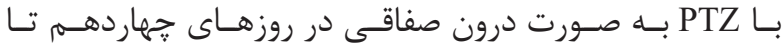

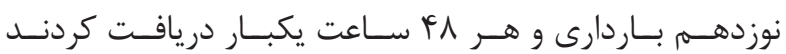

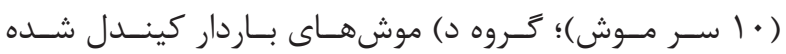

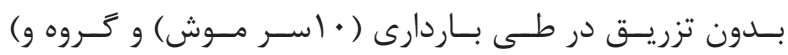

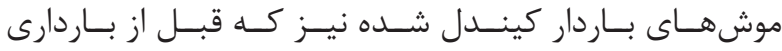

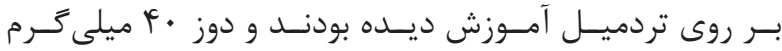

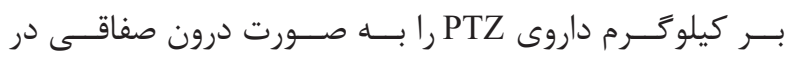

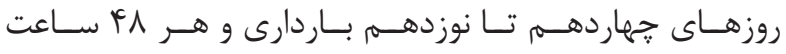

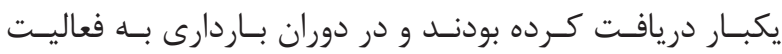

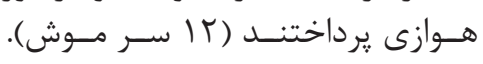

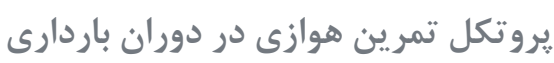

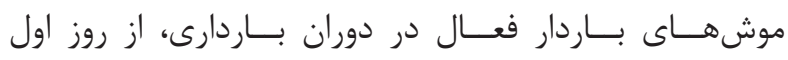

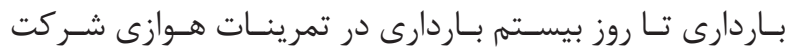

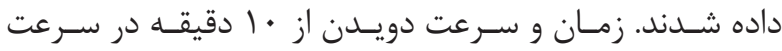

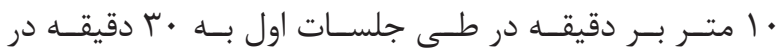

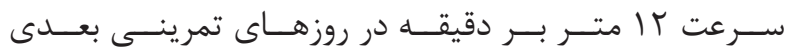

\footnotetext{
${ }^{4}$ Vaginal plug

${ }^{5}$ Intraperitoneal

${ }^{6}$ Forced swim test
} 


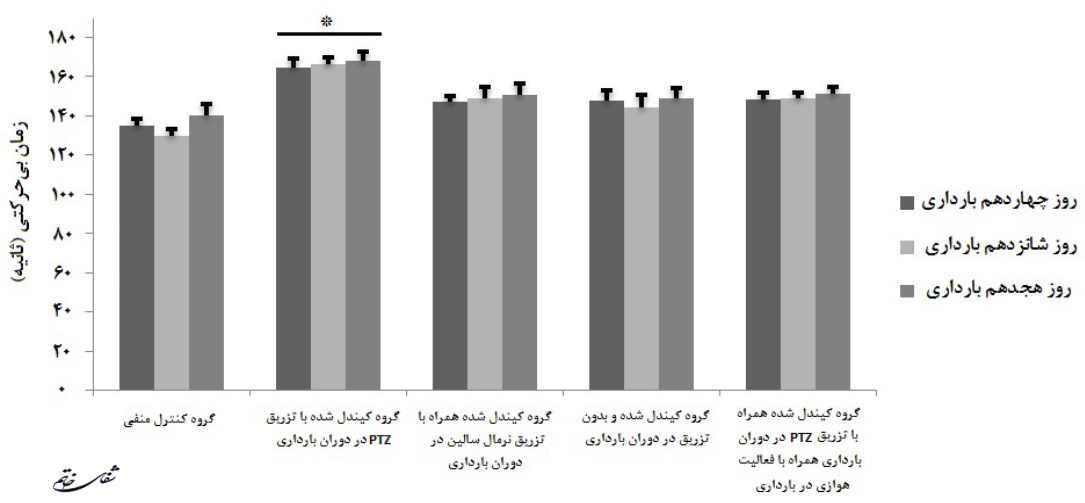

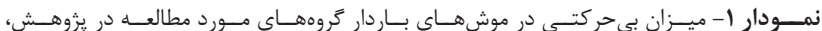

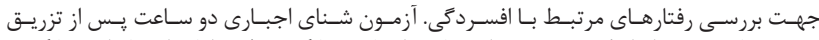

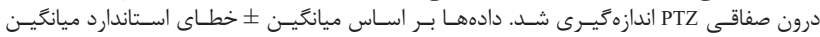

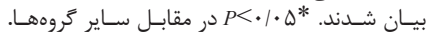

$$
\text { بحث و نتيجه }
$$

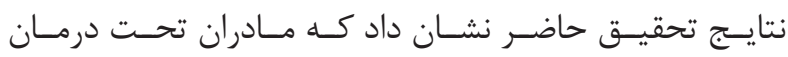

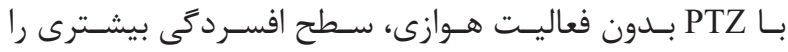

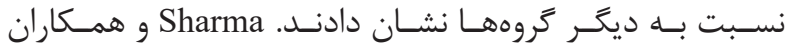

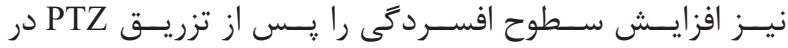

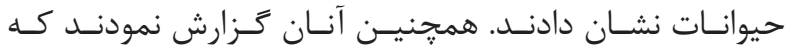

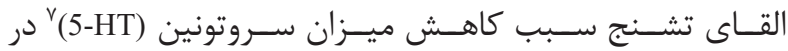

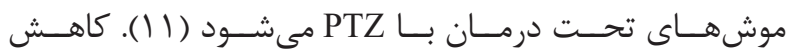

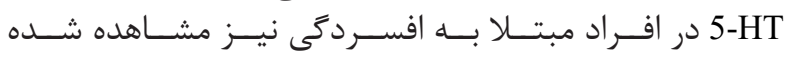

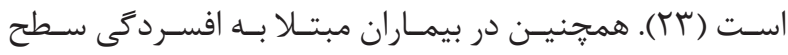

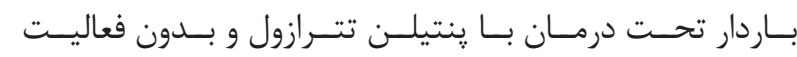

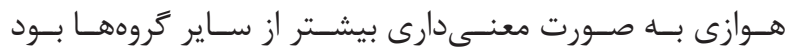

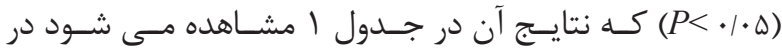

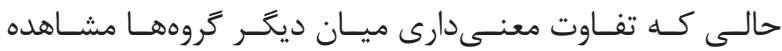

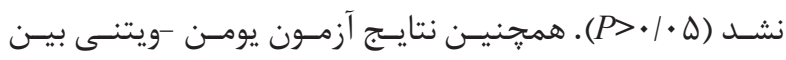

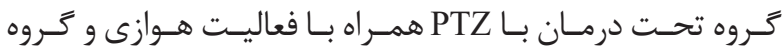

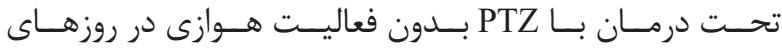

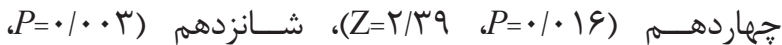
r

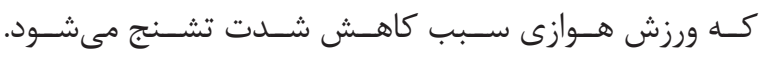

جدول ا- نتايج آزمون تعقيبى توكى جهت بررسى تفاوت بين كروهى حاصل از نتايج آزمون افسردگى.

\begin{tabular}{|c|c|c|c|c|}
\hline كروه كنترل منفى & كروه تحت درمان با نرمال سالين & با بعاليت هوازى درمان با PTZ & بدون فحت درماليت جسمانى با PTZ & كروههاى مورد مطالعه \\
\hline- & $P=\cdot / 119$ & $P=\cdot / \cdot$ 人. & $P<\cdot|\cdots \cdot|$ & كروه كنترل منفى \\
\hline$P=\cdot / \cdot \wedge$. & $P=1 / \cdots$ & $P=\cdot / \cdot r F$ & - & 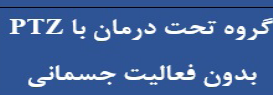 \\
\hline$P=\cdot / \mu \ldots$ & $P=1 / \ldots$ & $P=1 / \ldots$ & $P=\cdot / \cdot 11$ & كروه بدون تزريق در \\
\hline
\end{tabular}

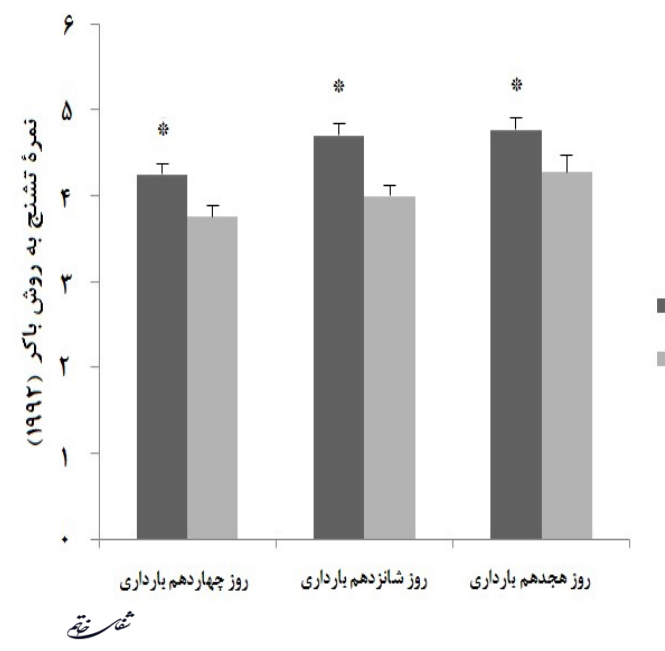

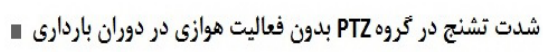

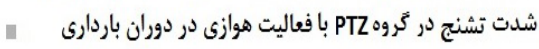

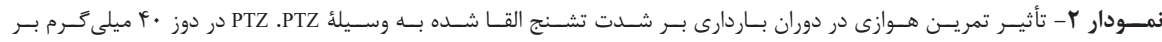

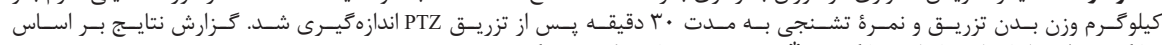

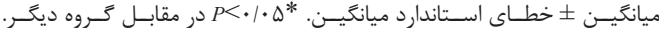

${ }^{7}$ Serotonin $(5-\mathrm{HT})$ 


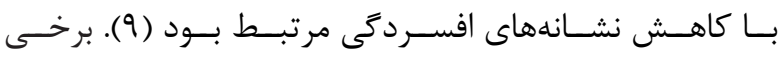

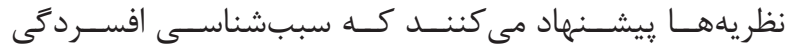

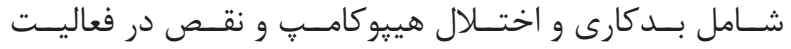

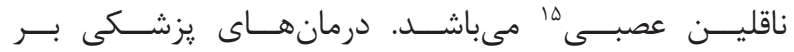

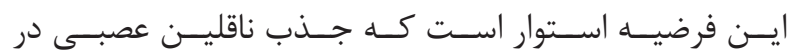

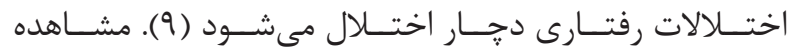

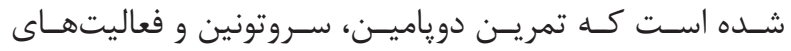

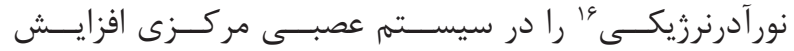

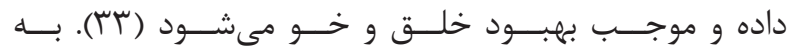

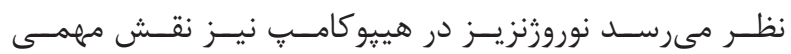

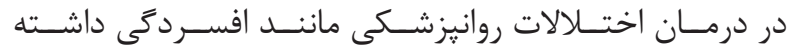

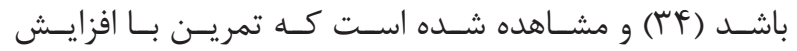

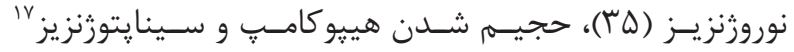

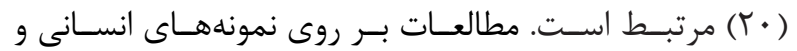

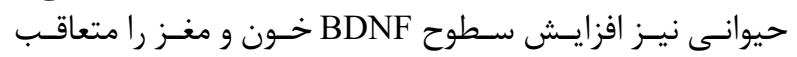

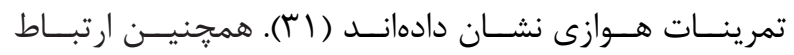

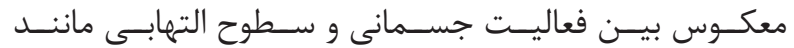

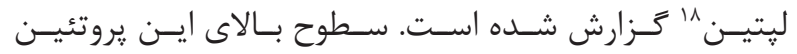

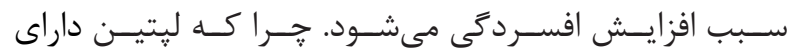

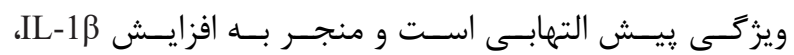
IL-6 و TNF-

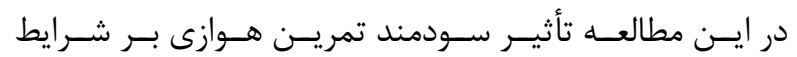

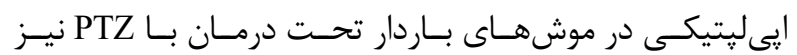

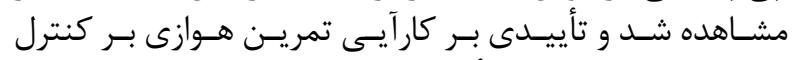

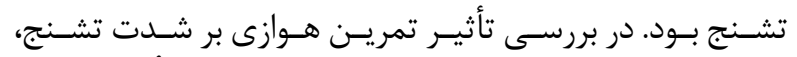

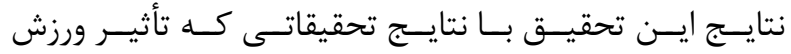

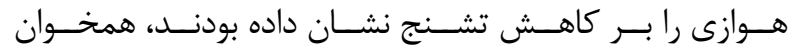

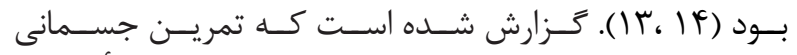

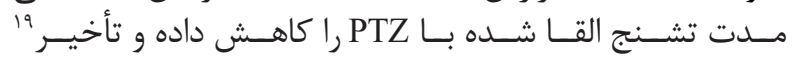

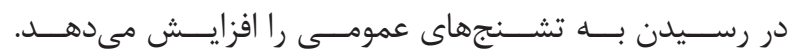

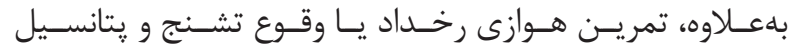

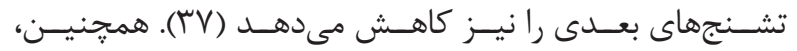

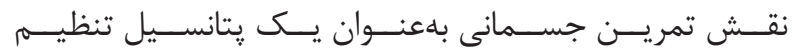

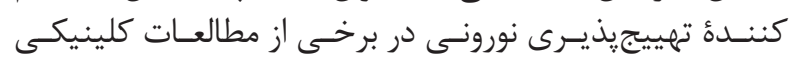

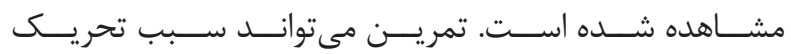

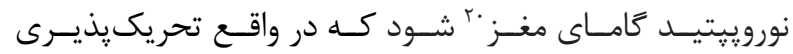

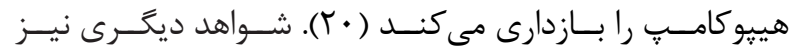

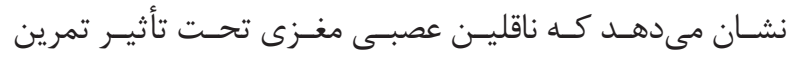

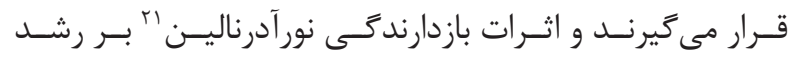

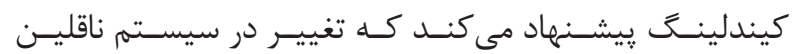

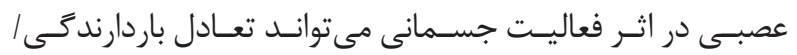

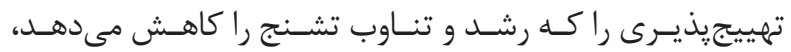

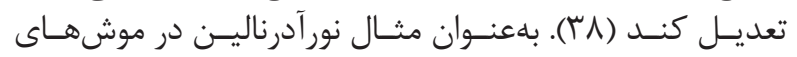

\footnotetext{
${ }^{8}$ L- Tryptophan

${ }^{9}$ Interleukin- $1 \beta$

${ }^{10}$ Interleukin-6

${ }^{11}$ Tumor necrosis factor alpha

${ }^{12}$ Hypothalamic-pituitary-adrenal axis

${ }^{13}$ Brain-derived neurotrophic factor

${ }^{14}$ Neurogenesis
}

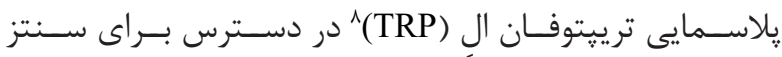

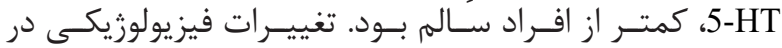

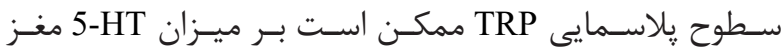

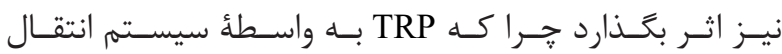

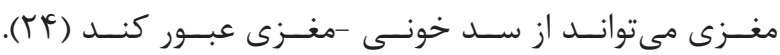

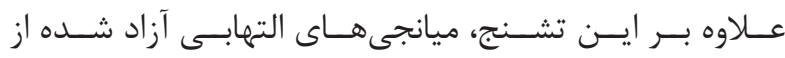

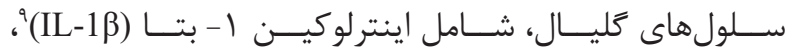

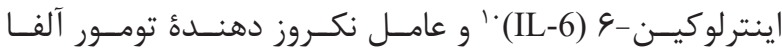

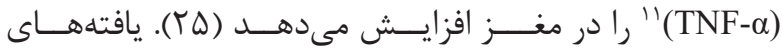

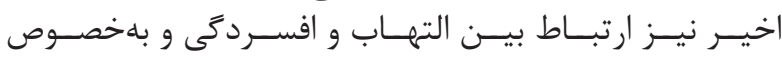

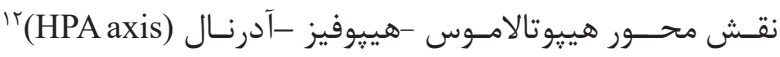

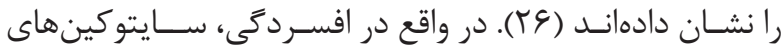

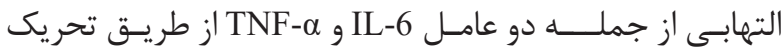

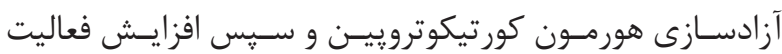

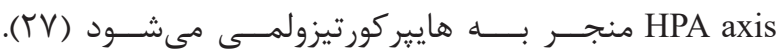

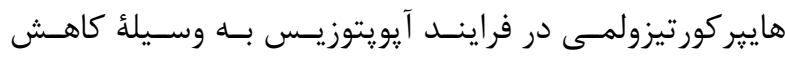

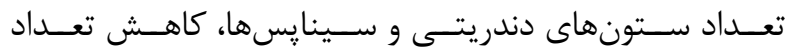

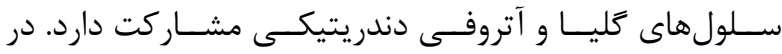

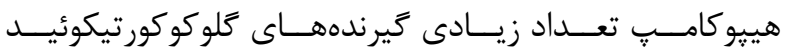

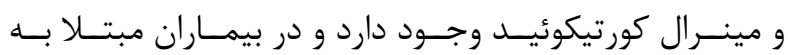

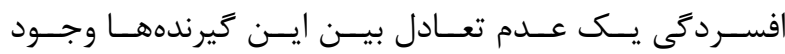

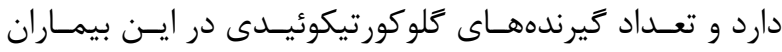

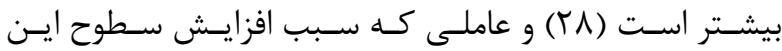

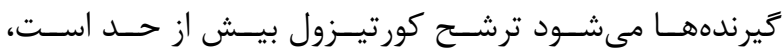

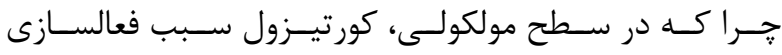

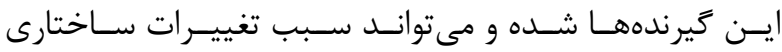

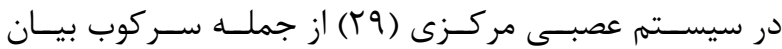

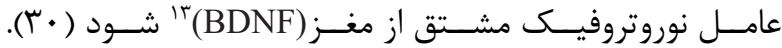

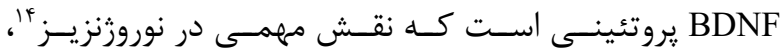

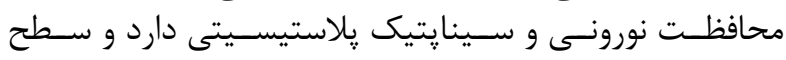

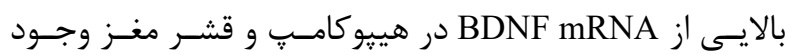

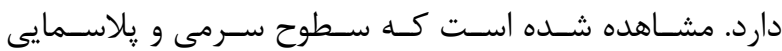

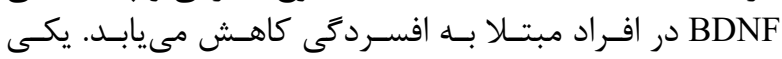

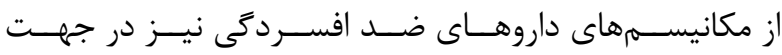

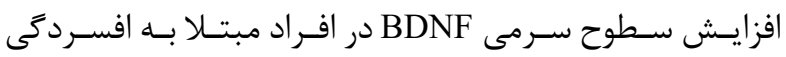

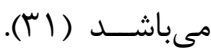

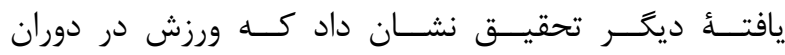

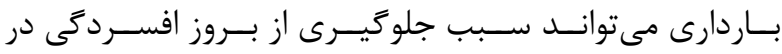

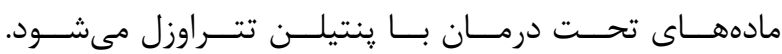

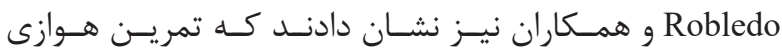

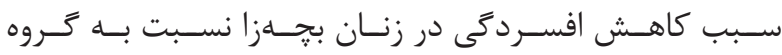

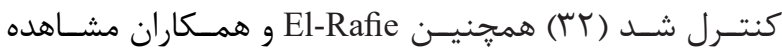

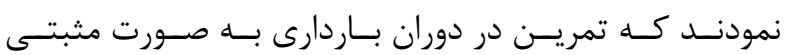

\footnotetext{
${ }^{15}$ Neurotransmitter

${ }^{16}$ Noradrenergic

${ }^{17}$ Synaptogenesis

${ }^{18}$ Leptin

${ }^{19}$ Latency

${ }^{20}$ Brain neuropeptide $\mathrm{Y}$

${ }^{21}$ Inhibitory influence of noradrenaline
} 


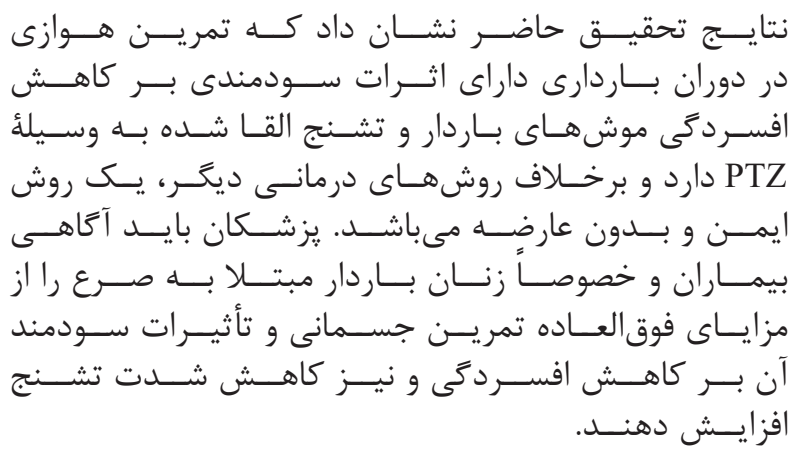

1. Fisher RS, Acevedo C, Arzimanoglou A, Bogacz A, Cross JH, Elger CE, et al. ILAE official report: a practical clinical definition of epilepsy. Epilepsia. 2014; 55(4): 475-82.

2. Ablah E, Hesdorffer DC, Liu Y, Paschal AM, Hawley $\mathrm{S}$, Thurman D, et al. Prevalence of epilepsy in rural Kansas. Epilepsy Res. 2014; 108(4): 792-801.

3. Novakova B, Harris PR, Reuber M. Diurnal patterns and relationships between physiological and selfreported stress in patients with epilepsy and psychogenic non-epileptic seizures. Epilepsy Behav. 2017; 70(Pt A): 204-11.

4. Borthen I, Gilhus NE. Pregnancy complications in patients with epilepsy. Curr Opin Obstet Gynecol. 2012; 24(2): 78-83.

5. Bjørk MH , Veiby G, Reiter SC, Berle JØ, Daltveit AK, Spigset O, et al. Depression and anxiety in women with epilepsy during pregnancy and after delivery: a prospective population-based cohort study on frequency, risk factors, medication ,andprognosis .Epilepsia. 2015; 56(1): 28-39.

6. Reiter SF, Veiby G, Daltveit AK, Engelsen BA, Gilhus NE. Psychiatric comorbidity and social aspects in pregnant women with epilepsy-the Norwegian Mother and Child Cohort Study. Epilepsy Behav. 2013; 29(2): $379-85$.

7. Herba CM, Glover V, Ramchandani PG, Rondon MB. Maternal depression and mental health in early childhood: an examination of underlying mechanisms in low-income and middleincome countries. Lancet Psychiatry 2016; 3(10): 983-92.

8. Jarde A, Morais M, Kingston D, et al. Neonatal outcomes in women with untreated antenatal depression compared with women without depression: a systematic review and metaanalysis. JAMA Psychiatry. 2016; 73(8): 826-37.

9. El-Rafie MM, Khafagy GM, Gamal MG. Effect

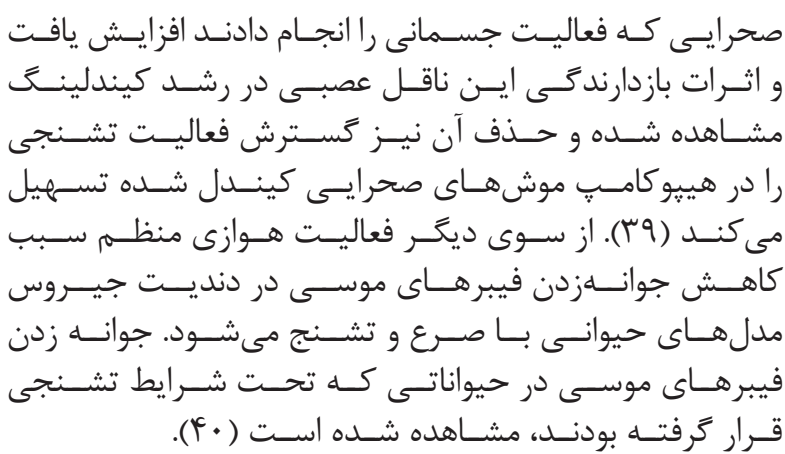

منابع

of aerobic exercise during pregnancy on antenatal depression. Int J Womens Health; 2016; 8: 53-7.

10. Kornum JB, Nielsen RB, Pedersen L, Mortensen PB, Nørgaard M. Use of selective serotonin-reuptake inhibitors during early pregnancy and risk of congenital malformations: updated analysis. Clin Epidemiol. 2010; 2: $29-36$.

11. Sharma RK, Singh T, Mishra A, Goel RK. Relative safety of different antidepressants for treatment of depression in chronic epileptic animals associated with depression. J Epilepsy Res. 2017; 7(1): 25-32.

12. Josefsson T, Lindwall M, Archer T. Physical exercise intervention in depressive disorders: Metaanalysis and systematic review." Scand J Med Sci Sports. 2014; 24(2): 259-72.

13. De Almeida AA, Gomes da Silva S, Lopim GM, Vannucci Campos D, Fernandes J, Cabral FR, et al. Resistance exercise reduces seizure occurrence, attenuates memory deficits and restores bdnf signaling in rats with chronic epilepsy. Neurochem Res. 2017; 42(4): 1230-9.

14. Hrncic D, Rasic-Markovic A, Lekovic J, Krstic D, Colovic M, MacutD, et al. Exercise decreases susceptibility to homocysteine seizures: the role of oxidative stress. Int J Sports Med. 2014; 35: 544-50.

15. Zhang B, Zhang J-W, Wang W-P, Dong R-F, Tian $S$, Zhang C. Effect of lamotrigine on epilepsyinduced cognitive impairment and hippocampal neuronal apoptosis in pentylenetetrazole-kindled animal model. Synapse. 2017; 71(2): doi: 10.1002/syn.21945.

16. Li B, Wang L, Sun Z, Zhou Y, Shao D, Zhao $\mathrm{J}$, et al. The anticonvulsant effects of SR 57227 on pentylenetetrazole-induced seizure in mice. PLoS One. 2014; 9(4): e93158. doi: 10.1371/journal.pone.

17. Pourmotabbed A, Nedaei SE, Cheraghi M, Moradian S, Touhidi A, Aeinfar M, et al. Effect of prenatal pentylenetetrazol-induced kindling on learning and 


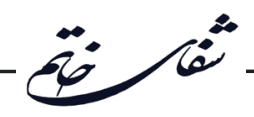

memory of male offspring. Neuroscience. 2011; 172: 205-11.

18. Zybura-Broda K, Amborska R, AmbrozekLatecka M, Wilemska J, Bogusz A, Bucko J, et al. Epigenetics of epileptogenesis-evoked upregulation of matrix metalloproteinase-9 in hippocampus. PLoS One. 2016; 11(8): e0159745. doi: 10.1371/journal.pone.

19. Rajabzadeh A, Bideskan AE, Fazel A, Sankian M, Rafatpanah H, Haghir H. The effect of PTZ-induced epileptic seizures on hippocampal expression of PSANCAM in offspring born to kindled rats. J Biomed Sci. 2012;19: 56. doi: 10.1186/1423-0127-19-56.

20. Gomes FG, Gomes Da Silva S, Cavalheiro EA, Arida RM. Beneficial influence of physical exercise following status epilepticus in the immature brain of rats. Neuroscience. 2014; 274: 69-81.

21. Majidi-Zolbanin J, Doosti MH, Kosari-Nasab M, Salari AA. Prenatal maternal immune activation increases anxiety- and depressive-like behaviors in offspring with experimental autoimmune encephalomyelitis. Neuroscience. 2015; 294: 69-81.

22. Gomes da Silva S, de Almeida AA, Fernandes J, Lopim GM, Cabral FR, Scerni DA, et al. Maternal exercise during pregnancy increases bdnf levels and cell numbers in the hippocampal formation but not in the cerebral cortex of adult rat offspring. PloS One. 2016; 11(1): e0147200. doi: 10.1371/journal.pone.

23. Patten SB. Medical models and metaphors for depression. Epidemiol Psychiatr Sci .2015; 24(4): 303-8.

24. Wichers MC, Koek GH, Robaeys G, Verkerk R, Scharpé S, Maes M. IDO and interferon-alpha-induced depressive symptoms: a shift in hypothesis from tryptophan depletion to neurotoxicity. Mol Psychiatry. 2005; 10(6): 538-44.

25. Vezzani A. Epilepsy and inflammation in the brain: overview and pathophysiology. Epilepsy Curr. 2014; 14(2): 3-7.

26. Furtado M, Katzman MA. Examining the role of neuroinflammation in major depression. Psychiatry Res. 2015; 229(1-2): 27-36.

27. McKay MS, Zakzanis KK. The impact of treatment on HPA axis activity in unipolar major depression. J Psychiatr Res. 2010; 44(3): 183-92.

28. Gianluca S. Neuroplasticity and major depression, the role of modern antidepressant drugs. World J Psychiatry. 2012; 2(3): 49-57.
29. Sousa N, Cerqueira JJ, Almeida OF. Corticosteroid receptors and neuroplasticity. Brain Res Rev. 2008; 57 : 561-70.

30. Chen H, Lombès M, Le Menuet D. Glucocorticoid receptor represses brain-derived neurotrophic factor expression in neuron-like cells. Mol Brain. 2017; 10(1): 12. doi: 10.1186/s13041-017-0295-x.

31. Kerling A, Kück M, Tegtbur U, Grams L, WeberSpickschen S, Hanke A. Exercise increases serum brain-derived neurotrophic factor in patients with major depressive disorder. J Affect Disord. 2017; 215: 152-5.

32. Robledo-Colonia AF, Sandoval-Restrepo N, Mosquera-Valderrama YF, et al. Aerobic exercise training during pregnancy reduces depressive symptoms in nulliparous women: a randomized trial. J Physiother. 2012; 58(1): 9-15.

33. Ploughman M. Exercise is brain food: the effects of physical activity on cognitive function. Dev Neurorehabil. 2008; 11(3): 236-40.

34. Nestler EJ, Barrot M, DiLeone RJ, Eisch AJ, Gold SJ, Monteggia LM. Neurobiology of depression. Neuron. 2002; 34(1): 13-25.

35. Nokia M, Lensu S, Ahtiainen J, Johansson PP, Koch LG, Britton SL, et al. Physical exercise increases adult hippocampal neurogenesis in male rats provided it is aerobic and sustained. J Physiol. 2016; 594 (7): 1855-73.

36. Abella V, Scotece M, Conde J, Pino J, GonzalezGay MA, Gómez-Reino JJ. Leptin in the interplay of inflammation, metabolism and immune system disorders. Nat Rev Rheumatol. 2017; 13(2): 100-9.

37. Kim, H, Kim IK, Song W. Lee J, Park S. The synergic effect of regular exercise and resveratrol on kainate-induced oxidative stress and seizure activity in mice. Neurochem Res. 2013; 38(1): 117-22.

38. Arida RM, de Almeida AC, Cavalheiro EA, Scorza FA. Experimental and clinical findings from physical exercise as complementary therapy for epilepsy. Epilepsy Behav. 2013; 26(3): 273-8.

39. Arida RM, Cavalheiro EA, Silva AC, Scorza FA. Physical activity and epilepsy: proven and predicted benefits. Sports Med. 2008; 38: 607-15.

40. Vannucci Campos D, Lopim GM, da Silva DA, de Almeida AA, Amado D, Arida RM. Epilepsy and exercise: an experimental study in female rats. Physiol Behav. 2017; 15(171): 120-6. 